\title{
Krukenberg Tumor in Adolescent: A Rare Occurrence
}

\author{
${ }^{1} \mathrm{G}$ Nandini, ${ }^{2} \mathrm{~K}$ Umadevi, ${ }^{3} \mathrm{~K}$ Padma, ${ }^{4}$ Rajini Uday
}

\begin{abstract}
Krukenberg tumors classically refer to secondary ovarian malignancy. The primary is mostly from female genital tract, breast and gastrointestinal tract. Krukenberg tumors account for 30 to $40 \%$ of metastatic cancers to the ovaries. The average age of occurrence is 45 years. Most of the Krukenberg tumors are histopathological diagnosis done postoperatively. We do report a case of Krukenberg tumor in an adolescent girl.
\end{abstract}

Keywords: Krukenberg, Metastatic, Signet ring, Bilateral.

How to cite this article: Nandini G, Umadevi K, Padma K, Uday R. Krukenberg Tumor in Adolescent: A Rare Occurrence. J South Asian Feder Obst Gynae 2014;6(3):202-204.

\section{Source of support: Nil}

Conflict of interest: None

\section{INTRODUCTION}

Malignant ovarian tumors may be primary or secondary. About $15 \%$ are metastatic and Krukenberg tumors constitute 30 to $40 \%$. They are usually bilateral and the presence of signet ring cell is very characteristic. In adolescent, majority of malignant tumors are of germ cell origin and metastatic Krukenberg tumor is very rare. Early diagnosis and treatment is paramount because of its poor prognosis.

\section{CASE REPORT}

Ms D, aged 17 years, presented with history of increased fatiguability, loss of weight and loss of appetite of 3 months duration. She also gave history of distension of abdomen, pain abdomen and vomiting from the past 2 months.

Her menstrual history was regular with 3/30 day cycle. Her last menstrual period was 20 days back. She was not married and not sexually active. Her history was nothing significant. Her family history was nothing contributing except for the fact, that her grandfather died of pulmonary Koch's 15 years prior.

\footnotetext{
${ }^{1,3,4}$ Professor, ${ }^{2}$ Ex-Professor

${ }^{1-4}$ Department of Obstetrics and Gynecology, MS Ramaiah Medical College, Bengaluru, Karnataka, India
}

Corresponding Author: G Nandini, Professor, Department of Obstetrics and Gynecology, MS Ramaiah Medical College, Bengaluru, Karnataka, India, Phone: 08023624573 +919480059850, e-mail: nandini_dr@yahoo.com
She had gone to a local hospital 2 months back where ascitic fluid tap was done and was started on anti Koch's treatment empirically.

On examination, she was moderately built and poorly nourished. Her vitals were stable except for mild pallor. Cervical lymph nodes were palpable. Per abdomen examination revealed uniform distension. Fluid thrill was present and there was no organomegaly. Per speculum and per vaginal examination was not done. Per rectal examination revealed nodularity and indurated mass anterior to rectum.

On ultrasonography (USG), both ovaries enlarged with heterogenous echotexture containing anechoic structures. Marked vascularity is seen in both the ovaries. Right ovary measured $6.8 \times 3.7 \mathrm{~cm}$. Left ovary measured $7.5 \times 5.2 \mathrm{~cm}$. Gross free fluid in the abdomen and pelvis with low level internal echoes. Uterus was normal and ET was $4 \mathrm{~mm}$.

Tumor markers were done. Her CA-125 was $94.7 \mathrm{IU} / \mathrm{ml}$. Serum AFP and Beta hCG was normal.

Computed tomography (CT) scan showed heterogeneously enhancing mass in bilateral adnexa measuring $60 \times 42 \times 43 \mathrm{~mm}$ on right side and $83 \times 52 \times 40 \mathrm{~mm}$ on left side. The ovaries were not separately visualized. Both lesions showed well enhancing peripheral rim and a central heterogeneously enhancing area. No calcification or necrotic area seen. Enhancing and thick omentum noted. Moderate to gross free fluid in the peritoneal cavity.

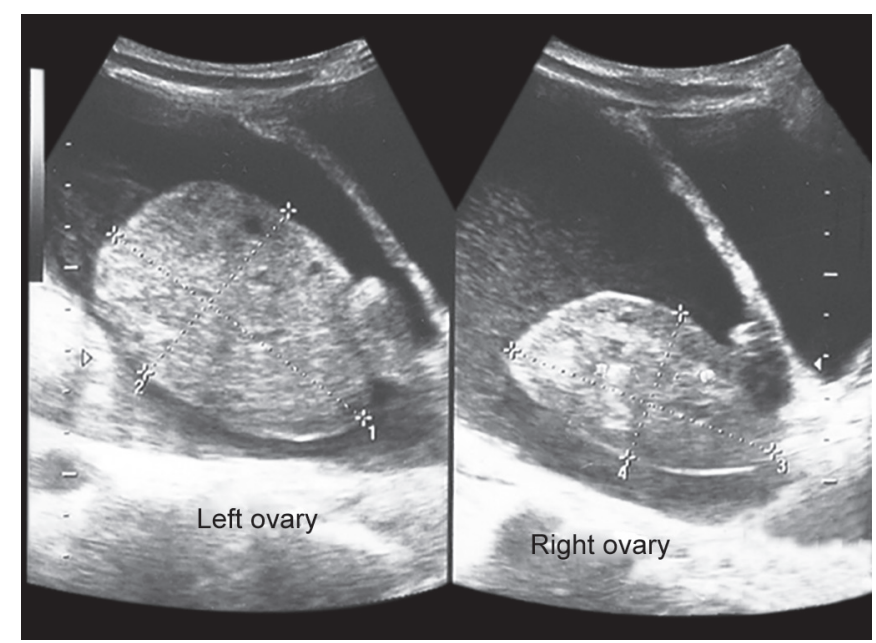

Fig. 1: Ultrasound of abdomen showing bilateral large ovarian masses with heterogenous echotexture. Marked vascularitis seen (Source: MS Ramaiah Hospital, Bengaluru, Karnataka, India) 


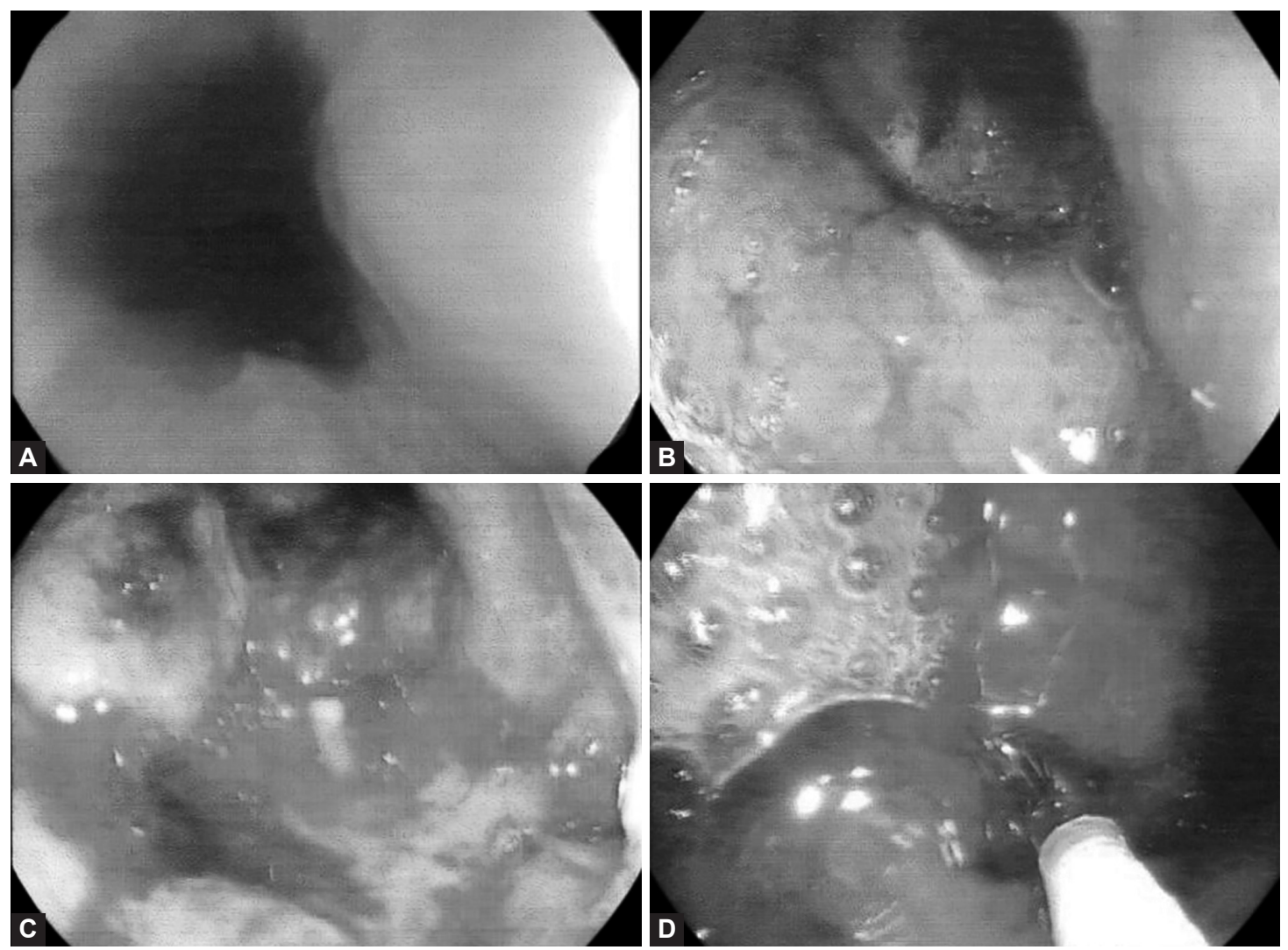

Figs 2A to D: (A) Gastroesophageal junction, (B and C) stomach showing thickened and edematous folds with poor distensibility and (D) taking biopsy (Source: Endoscopy, Department of Gastroenterology, MS Ramaiah Hospital, Bengaluru, Karnataka, India)

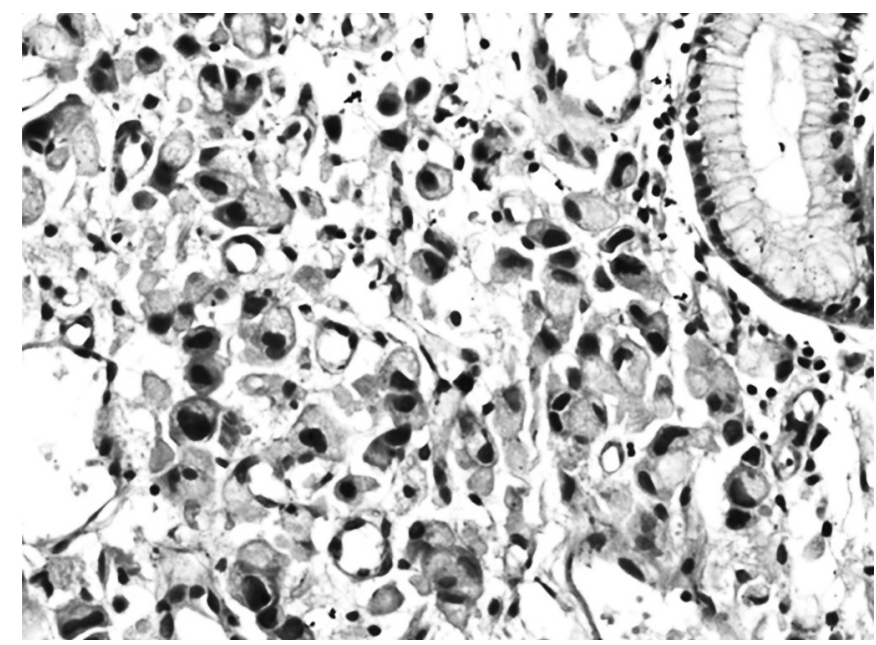

Fig. 3: Histopathology of biopsy from gastric growth showing diffuse infiltration and compromised of discohesive, moderately pleomorphic round to polygonal cells with anisonucleosis, hyperchromatism, nucleomegaly. Many cells have a signet ring appearance with eccentrically disposed nucleus and intracytoplasmic mucin vacuole. Features of signet cell carcinoma. (Source: Department of Pathology, MS Ramaiah Hospital, Bengaluru, Karnataka, India)

Our probable diagnosis was Krukenberg tumor. The endoscopy findings were as follows:

- Esophagus: Normal mucosa. No hiatus hernia.

- Stomach: Ulcerated growth noted extending from 38 to $42 \mathrm{~cm}$ with poor distensibility, normal pylorus and no stasis.
- Duodenum: Normal D1 and D2 mucosa. Probability of gastric carcinoma and biopsy was taken.

\section{HISTOPATHOLOGY}

Gastric antral mucosa diffusely infiltrated by tumor.

Discohesive, round to polygonal cells. Anisonucleosis, intracytoplasmic mucin. Majority: signet ring appearanceeccentric disposed nucleus and intracytoplasmic vacuolesuggesting signet ring cell carcinoma.

Fine needle aspiration cytology (FNAC) of cervical lymph node was positive for malignancy, and ascitic fluid cytology was suggesting signet ring cell carcinoma.

- Diagnosis: Endoscopy - gastric carcinoma

- HPE: Signet cell carcinoma

- USG and CT: Krukenberg tumor

- Ascitic fluid cytology: Signet cell carcinoma

Final diagnosis was signet ring cell carcinoma stomach stage IV.

Planned for multimodality approach. First was to give chemotherapy, then surgical treatment. She was planned for palliative chemotherapy with OLF CT. She received two cycles of oxaliplatin, leucoverin and 5 fluorouracil. Due to increase in ascitis and persistent progressive disease therapy was modified and changed. Paracentesis done and 3 liters of fluid drained. Therapy changed to irinotecan and oxaliplatin. After two cycles of chemo- 
therapy, disease progressed. About 3 liters of fluid drained through paracentesis and patient succumbed to the disease.

\section{DISCUSSION}

Krukenberg tumors mostly occur after 40 years metastatic ovarian tumors in young are very rare. In adolescent, it is usually germ cell tumors. Krukenberg tumor is metastasis mainly from gastric carcinoma in two-third of the cases. ${ }^{1}$ The other sites in order of frequency are appendix, colon, breast, small intestine, rectum, gallbladder and urinary bladder. ${ }^{1}$ To differentiate primary ovarian mucinous carcinoma from metastatic carcinoma is that primary is usually unilateral tumor and absence of signet ring cells. ${ }^{3}$

Management must involve finding and treating the primary tumor. Multimodality approach is ideal. The lesions are usually not discovered until the primary disease is advanced, and therefore most patients die of their disease within 1 year. In some cases, a primary tumor is never found. Some authorities advocate bilateral oophorectomy during surgery. Prognosis is poor. ${ }^{2}$ Radical surgery if solitary metastasis. Otherwise chemotherapy and radiotherapy to shrink the tumor and then plan for surgery.

\section{CONCLUSION}

Though rare, can occur in young. Early diagnosis may help in survival.

\section{REFERENCES}

1. Kiyokawa T, Young RH, Scully RE. Krukenberg tumors of the ovary: a clinicopathologic analysis of 120 cases with emphasis on their variable pathologic manifestations. Am J Surg Patho 2006 Mar;30(3):277-299.

2. Sanchez Lihon J. Krukenberg ovary tumor pathological clinical study of 56 cases in the Instituto Nacional de Enfermedades Neplasicas (National Cancer Institute) (Article in Spanish). Rev Gastroenterol Peru 2009 Jul-Sep;29(3):209-217.

3. Hatwal D, Joshi C, Chaudhari S, Bhatt P. Krukenberg tumor in a young woman: a rare presentation. Ind J Patho Microbiol 2014;57(1):124-126 